FRACTIONAL CALCULUS

AN IntRodUCtion For PHYSICISTS 
This page intentionally left blank 


\section{FRACTIONAL CALCULUS}

AN INTRODUCTION FOR PHYSICISTS

\section{RICHARD HERRMANN}

GigaHedron, Germany 
Published by

World Scientific Publishing Co. Pte. Ltd.

5 Toh Tuck Link, Singapore 596224

USA office: 27 Warren Street, Suite 401-402, Hackensack, NJ 07601

UK office: 57 Shelton Street, Covent Garden, London WC2H 9HE

\section{British Library Cataloguing-in-Publication Data}

A catalogue record for this book is available from the British Library.

\section{FRACTIONAL CALCULUS \\ An Introduction for Physicists}

Copyright (C) 2011 by World Scientific Publishing Co. Pte. Ltd.

All rights reserved. This book, or parts thereof, may not be reproduced in any form or by any means, electronic or mechanical, including photocopying, recording or any information storage and retrieval system now known or to be invented, without written permission from the Publisher.

For photocopying of material in this volume, please pay a copying fee through the Copyright Clearance Center, Inc., 222 Rosewood Drive, Danvers, MA 01923, USA. In this case permission to photocopy is not required from the publisher.

ISBN-13 978-981-4340-24-3

ISBN-10 981-4340-24-3

Printed in Singapore. 


\section{Foreword}

Theoretical physics has evolved into a multi-faceted science. The sheer amount of knowledge accumulated over centuries forces a lecturer to focus on the mere presentation of derived results. The presentation of strategies or the long path to find appropriate tools and methods often has been neglected.

As a consequence, from a student's point of view, theoretical physics seems to be a construct of axiomatic completeness, where apparently is no space left for speculations and new approaches. The limitations of presented concepts and strategies are not obvious until these tools are applied to new problems.

Especially the creative process of research, which evolves beyond the state of mere reception of already known interrelations is a particular ambition of most students.

One prerequisite to reach that goal is a permanent readiness, to question even well-established results and check the validity of common statements time and time again. Already Roger Bacon in his opus majus pointed out, that the main causes of error are the belief in false authority, the force of habit, the ignorance of others and pretended knowledge [Bacon(1267)].

New concepts and new methods depend on each other. The unified theory of electromagnetism by James Clerk Maxwell was motivated by Faraday's experiments. The definite formulation was then realized in terms of a new theory of partial differential equations. Einstein's general relativity is based on experiments on the constancy of the speed of light; the elegant presentation was made possible with Riemann's tensor calculus.

This book too takes up an idea which is simple at first: due to the fact that differential equations play such a central role in physics since the times of Newton and since the first and second derivatives denote such 
fundamental properties as velocity and acceleration, does it make sense, to investigate the physical meaning of a half, a $\pi$-th and an imaginary derivative?

This interesting question has been raised since the days of Leibniz and has been discussed by all mathematicians of their times. But for centuries no practical applications could be imagined.

During the last years fractional calculus has developed rapidly. This process is still going on, but we can already recognize, that within the framework of fractional calculus new concepts and strategies emerge, which make it possible, to obtain new challenging insights and surprising correlations between different branches of physics.

This is the basic purpose of this book: to present a concise introduction to the basic methods and strategies in fractional calculus and to enable the reader to catch up with the state of the art on this field and to participate and contribute in the development of this exciting research area.

In contrast to other monographs on this subject, which mainly deal with the mathematical foundations of fractional calculus, this book is devoted to the application of fractional calculus on physical problems. The fractional concept is applied to subjects in classical mechanics, group theory, quantum mechanics, nuclear physics, hadron spectroscopy up to quantum field theory and will surprise the reader with new intriguing insights.

This book provides a skillful insight into a vividly growing research area and opens the reader's mind to a world yet completely unexplored. It encourages the reader to participate in the exciting quest for new horizons of knowledge.

Richard Herrmann

Dreieich, Germany

Summer, 2010 


\section{Acknowledgments}

As a young student, I had the opportunity to attend the Nobel Laureate Meeting at Lindau/Germany in 1982. Especially Paul Dirac's talk was impressive and highly inspiring and it affirmed my aspiration to focus my studies on theoretical physics.

I joined the Institute for Theoretical Physics in Frankfurt/Germany. Working on the fission properties of super-heavy elements, I suggested to linearise the collective Schrödinger equation used in nuclear collective models and to introduce a new collective degree of freedom, the collective spin. Within this project, my research activities are concentrated on the field theoretical implications, which follow from linearised wave equations. As a direct consequence the question of the physical interpretation of multifactorized wave equations and of a fractional derivative came up.

I achieved a breakthrough in my research program in 2005: I found that a specific realization of the fractional derivative suffices to describe the properties of a fractional extension of the standard rotation group $S O(n)$. This was the key result to start an investigation of symmetries of fractional wave equations and the concept of a fractional group theory could be successfully realized. Up to now, this concept has led to a vast amount of intriguing and valuable results.

This success would not have been possible without the permanent enduring loving support of my family and friends.

I am grateful to all those who shared their knowledge with me and made suggestions reflected in this book. Special thanks go to Anke Friedrich and Günter Plunien for their encouragement and their valuable contributions.

I also want to emphasize, that fractional calculus is a worldwide activity. I benefited immensely from international support and communication. Discussions and correspondence with Eberhard Engel, Ervin Goldfain, Cesar 
Ionescu, Swee Cheng Lim, Ahmad-Rami El-Nabulsi, Manuel Ortigueira and Volker Schneider were particularly useful.

Finally I want to express my gratitude to the publishing team of Word Scientific for their support. 


\section{Contents}

Foreword $\quad$ v

Acknowledgments vii

1. Introduction 1

2. Functions 5

2.1 Gamma function . . . . . . . . . . . . . 6

2.2 Mittag-Leffler functions . . . . . . . . . . . . . . . . 7

2.3 Hypergeometric functions . . . . . . . . . . . . . . . . . . 9

2.4 Miscellaneous functions . . . . . . . . . . . . . 9

3. The Fractional Derivative 11

$3.1 \quad$ Basics . . . . . . . . . . . . . . . . 11

3.2 The fractional Leibniz product rule . . . . . . . . . . 16

3.3 Discussion . . . . . . . . . . . . . . . . . 18

3.3.1 Orthogonal polynomials . . . . . . . . . . 18

3.3.2 Differential representation of the Riemann fractional derivative . . . . . . . . . . . 20

4. Friction Forces 23

4.1 Classical description . . . . . . . . . . . . . . . . . 23

4.2 Fractional friction . . . . . . . . . . . 26

5. Fractional Calculus 33

5.1 The Fourier transform . . . . . . . . . . . . . . . 34 
5.2 The fractional integral . . . . . . . . . . . . 35

5.2.1 The Liouville fractional integral . . . . . . . . 36

5.2.2 The Riemann fractional integral . . . . . . . . . 36

5.3 Correlation of fractional integration and differentiation . . 37

5.3.1 The Liouville fractional derivative . . . . . . . . 38

5.3.2 The Riemann fractional derivative . . . . . . . . 39

5.3.3 The Liouville fractional derivative with inverted operator sequence: the Liouville-Caputo fractional derivative ................ 40

5.3.4 The Riemann fractional derivative with inverted operator sequence: the Caputo fractional derivative 42

5.4 Fractional derivative of second order . . . . . . . . . 43

5.4.1 The Riesz fractional derivative . . . . . . . . . . 44

5.4 .2 The Feller fractional derivative . . . . . . . . . . 46

5.5 Fractional derivatives of higher orders . . . . . . . . 47

5.6 Geometric interpretation of the fractional integral . . . . 51

5.7 Low level fractionality . . . . . . . . . . . 53

5.8 Discussion . . . . . . . . . . . . . 55

5.8.1 Semigroup property of the fractional integral . . . 55

6. The Fractional Harmonic Oscillator $\quad 57$

6.1 The fractional harmonic oscillator . . . . . . . . 58

6.2 The harmonic oscillator according to Fourier . . . . . . 58

6.3 The harmonic oscillator according to Riemann . . . . . 60

6.4 The harmonic oscillator according to Caputo . . . . . . . 62

7. Wave Equations and Parity 65

7.1 Fractional wave equations . . . . . . . . . . . 65

7.2 Parity and time-reversal . . . . . . . . . . . . 67

7.3 Solutions of the free regularized fractional wave equation . 68

8. Nonlocality and Memory Effects 75

8.1 A short history of nonlocal concepts . . . . . . . . 75

8.2 From local to nonlocal operators . . . . . . . . . . 77

8.3 Memory effects . . . . . . . . . . . . . 88

9. Quantum Mechanics 93

9.1 Canonical quantization . . . . . . . . . . . . . 95 
9.2 Quantization of the classical Hamilton function and free

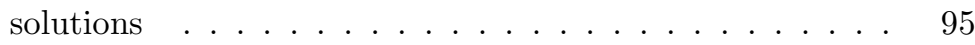

9.3 Temperature dependence of a fission yield and determination of the corresponding fission potential . . . . . . 99

9.4 The fractional Schrödinger equation with an infinite well potential . . . . . . . . . . . . . . 102

9.5 Radial solutions of the fractional Schrödinger equation . . 107

10. Fractional Spin: a Property of Particles Described with the Fractional Schrödinger Equation

10.1 Spin . . . . . . . . . . . . . . . . . . 112

10.2 Fractional spin . . . . . . . . . . . . . . 113

$\begin{array}{ll}\text { 11. Factorization } & 117\end{array}$

11.1 The Dirac equation . . . . . . . . . . . . . . . . 117

11.2 The fractional Dirac equation . . . . . . . . . . . . 118

11.3 The fractional Pauli equation . . . . . . . . . . . 120

12. Symmetries 123

12.1 Characteristics of fractional group theory . . . . . . . . 124

12.2 The fractional rotation group $\mathrm{SO}_{N}^{\alpha} \ldots \ldots \ldots \ldots 126$

13. The Fractional Symmetric Rigid Rotor 133

13.1 The spectrum of the fractional symmetric rigid rotor . . . 133

13.2 Rotational limit . . . . . . . . . . . . . . . . 136

13.3 Vibrational limit . . . . . . . . . . . . . . . . 137

13.4 Davidson potential: the so called $\gamma$-unstable limit . . . . 138

13.5 Linear potential limit . . . . . . . . . . . . . . . . . . 140

13.6 The magic limit . . . . . . . . . . . . . . . . . . . 141

13.7 Comparison with experimental data . . . . . . . . . 144

14. q-deformed Lie Algebras and Fractional Calculus 153

14.1 q-deformed Lie algebras . . . . . . . . . . . . . . 153

14.2 The fractional q-deformed harmonic oscillator . . . . . . 156

14.3 The fractional q-deformed symmetric rotor . . . . . . . 160

14.4 Half-integer representations of the fractional rotation group $S O^{\alpha}(3) \ldots \ldots \ldots \ldots \ldots \ldots$ 
15. Fractional Spectroscopy of Hadrons

15.1 Phenomenology of the baryon spectrum . . . . . . . 166

15.2 Charmonium . . . . . . . . . . . . . . . . 171

15.3 Phenomenology of meson spectra . . . . . . . . . . 176

15.4 Metaphysics: About the internal structure of quarks . . . 184

16. Higher Dimensional Fractional Rotation Groups 187

16.1 The four decompositions of the mixed fractional $\mathrm{SO}^{\alpha}(9)$. 187

16.2 Notation . . . . . . . . . . . . . . . . 189

16.3 The nine dimensional fractional

Caputo-Riemann-Riemann symmetric rotor . . . . . . . . 192

16.4 Magic numbers of nuclei . . . . . . . . . . . . . . . 193

16.5 Ground state properties of nuclei . . . . . . . . . 196

16.6 Fine structure of the single particle spectrum: the extended Caputo-Riemann-Riemann symmetric rotor . . . 201

16.7 Magic numbers of electronic clusters: the nine dimensional fractional Caputo-Caputo-Riemann symmetric rotor . . . 206

16.8 Binding energy of electronic clusters . . . . . . . . . . 210

16.9 Metaphysics: magic numbers for clusters bound by weak and gravitational forces respectively . . . . . . . . . 213

17. Fractors: Fractional Tensor Calculus 219

17.1 Covariance for fractional tensors . . . . . . . . . . 219

17.2 Singular fractional tensors . . . . . . . . . . . . 220

18. Fractional Fields 223

18.1 Fractional Euler-Lagrange equations . . . . . . . . . . . . 224

18.2 The fractional Maxwell equations . . . . . . . . . . . 227

19. Gauge Invariance in Fractional Field Theories 231

19.1 Gauge invariance in first order of the coupling constant $\bar{g} \quad 232$

19.2 The fractional Riemann-Liouville-Zeeman effect . . . . . 236

20. Outlook 241

Bibliography 243

$\begin{array}{ll}\text { Index } & 257\end{array}$ 